\title{
Striatal Dopamine Transporter Binding Does Not Correlate with Clinical Severity in Dementia with Lewy Bodies
}

\author{
Morten Ziebell ${ }^{1}$, Birgitte B. Andersen ${ }^{2}$, Lars H. Pinborg ${ }^{1,3}$, Gitte M. Knudsen ${ }^{1}$, Jette Stokholm ${ }^{2}$, Gerda Thomsen ${ }^{1}$, \\ Merete Karlsborg ${ }^{4}$, Peter Høgh ${ }^{5}$, Mette Louise Mørk ${ }^{1}$, and Steen G. Hasselbalch ${ }^{1,2}$ \\ ${ }^{I}$ Neurobiology Research Unit and Cimbi, Rigshospitalet and University of Copenhagen, Copenhagen, Denmark; ${ }^{2}$ Memory Disorders \\ Research Group, Department of Neurology, Rigshospitalet and University of Copenhagen, Copenhagen, Denmark; ${ }^{3}$ Epilepsy Clinic, \\ Department of Neurology, Rigshospitalet and University of Copenhagen, Copenhagen, Denmark; ${ }^{4}$ Clinic for Movement Disorders, \\ Department of Neurology, Bispebjerg Hospital, Copenhagen, Denmark; and ${ }^{5}$ Regional Dementia Research Centre, Department of \\ Neurology, Copenhagen University Hospital, Roskilde, Denmark
}

Patients who have dementia with Lewy bodies (DLB) show both clinical and histopathologic overlap with Alzheimer disease patients and Parkinson disease patients. In this study, we correlated the core features of DLB (dementia, parkinsonism, hallucinations, and fluctuations) with striatal dopamine transporter (DAT) availability as assessed with SPECT and ${ }^{123} \mathrm{I}-\mathrm{N}$-(3-iodoprop-2E-enyl)-2- $\beta$-carbomethoxy-3 $\beta$-(4-methylphenyl) nortropane (123|-PE2I) in patients with newly diagnosed DLB. Methods: Two hundred eighty-eight patients were consecutively included in the study as they were referred for diagnostic SPECT scanning of DAT with 123/-PE2I. Of those patients, 51 had, on the basis of clinical guideline criteria, a probable-DLB diagnosis at follow-up $16 \pm 11.6$ mo later. Before or on the day of the SPECT scan, DLB patients had a routine neurologic examination including Hoehn and Yahr grading and were cognitively evaluated with the Mini Mental State Examination. Results: There was no correlation between Mini Mental State Examination, Hoehn and Yahr score, fluctuations or hallucinations, and striatal DAT availability as measured with ${ }^{123}|-P E 2|$ and SPECT. Conclusion: In patients with newly diagnosed DLB, symptoms are not associated with a reduction in striatal DAT despite its firm involvement in DLB pathology.

Key Words: SPECT; dopamine transporter; DLB

J Nucl Med 2013; 54:1072-1076

DOI: 10.2967/jnumed.112.114025

$\mathbf{T}$ he central feature in dementia with Lewy bodies (DLB) is progressive dementia, but compared with other forms of dementia it has several additional frequent core features, namely fluctuations, hallucinations, and parkinsonism. Parkinson disease (PD), DLB, and PD dementia are seen as a spectrum of the same condition, "Lewy body diseases" (1). In the clinic, DLB and PD dementia are nosologically differentiated only by the time span between the development of parkinsonism and dementia. In DLB, cognitive problems occur less than a year after the onset of parkinsonism (2). The dopamine system is deeply involved in all

Received Sep. 14, 2012; revision accepted Jan. 28, 2013.

For correspondence contact: Morten Ziebell, Neurobiology Research Unit,

Rigshospitalet, N9201, 9 Blegdamsvej, Copenhagen, DK-2100 Denmark.

E-mail: ziebell@nru.dk

Published online May 1, 2013.

COPYRIGHT (C 2013 by the Society of Nuclear Medicine and Molecular Imaging, Inc.
Lewy body diseases, and dopamine is reduced in the basal ganglia as a consequence of the aggregation of Lewy bodies in this area of the brain $(3,4)$.

Imaging of the dopamine transporter (DAT) with SPECT serves as a surrogate marker of the nerve connection between axons from the substantia nigra and neurons in the striatum $(5,6)$. An in vitro study of brains from monkeys treated with 1-methyl-4-phenyl1,2,3,6-tetrahydropyridine, meant as a model of human DLB, suggested that the loss of DAT and the loss of striatal dopamine content are linearly correlated (7).

In PD, many studies have shown a clear correlation between DAT binding and core features of parkinsonism (8). We identified only one DAT SPECT imaging study including 15 DLB patients correlating DAT binding (in which ${ }^{123} \mathrm{I}-2-\beta$-carbomethoxy-3 $\beta$-(4iodophenyl)- $N$-(3-fluoropropyl)nortropane [123I-FP-CIT] was used as the radioligand) with parkinsonism. The authors suggested that there might be a difference in the predilection for DAT uptake in PD versus DLB, since the caudate-to-putamen ratio was less reduced in DLB than in PD and, further, no correlation between severity of parkinsonism and DAT binding was found in DLB patients (9).

SPECT imaging of cerebral blood flow has demonstrated frontoparietooccipital hypoperfusion patterns in DLB patients (10). The frontal hypoperfusion is interesting, since the dorsolateral circuit involved in planning and attention connects the frontal cortex with the striatum and thus attentional fluctuations are also greatly influenced by the striatum and the dopamine system (11). One study that included 18 patients with well-defined DLB and used ${ }^{123}$ I-FP-CIT as the radioligand found a correlation between SPECT DAT and visual hallucinations (12). However, this study would be interesting to replicate.

Thus, the aim of this study was to identify whether any of the core features of DLB (dementia, hallucinations, fluctuations, or parkinsonism) were influenced by disturbances in DAT availability. If this were the case, we would expect a correlation between clinical symptoms and striatal dopaminergic availability, assessed with SPECT and the highly DAT-selective radioligand ${ }^{123} \mathrm{I}-\mathrm{N}-(3-$ iodoprop-2E-enyl)-2- $\beta$-carbomethoxy-3 $\beta$-(4-methylphenyl) nortropane ( $\left.{ }^{123} \mathrm{I}-\mathrm{PE} 2 \mathrm{I}\right)(13)$.

\section{MATERIALS AND METHODS}

Patients and Healthy Controls

The DLB patients comprised a subgroup of a larger group of patients with movement disorders, as reported in a previous study (14). 
Two hundred eighty-eight patients were continuously enrolled from January 2003 until March 2008, as they were admitted for a diagnostic DAT SPECT scan to the Department of Neurology, Rigshospitalet, Copenhagen. At the time of the DAT SPECT scan, the patients accepted the examination by written consent.

In the same period, 28 healthy volunteers (16 men and 12 women; mean age, $47.8 \mathrm{y}$; range, 23-71 y) were scanned with DAT SPECT. The procedures were identical to the ones described earlier, with the diagnosis being finally established by clinical follow-up months later (15-17). The study was performed in accordance with the ethical standards of the Declarations of Helsinki and was approved by the ethical committee of Copenhagen (protocol KF 02-150/98, KF 12-009/04, H-B-2008-024).

\section{SPECT Scanning}

To block thyroidal uptake of free radioiodine, the subjects received $200 \mathrm{mg}$ of potassium perchloride intravenously 15-20 min before injection of the DAT radioligand ${ }^{123}$ I-PE2I. Subsequently, an intravenous bolus of ${ }^{123}$ I-PE2I was given, immediately followed by a constant infusion of ${ }^{123} \mathrm{I}$-PE2I for $3 \mathrm{~h}$ (15).

After $2 \mathrm{~h}$ of infusion, SPECT imaging was performed with a triplehead IRIX camera (Philips Medical) fitted with low-energy, generalpurpose, parallel-hole collimators (spatial resolution, $8.5 \mathrm{~mm}$ at 10 $\mathrm{cm}$ ). The images were reconstructed with a MATLAB 6.5 (MathWorks)-based program using standard filtered backprojection with a low-pass fourth-order Butterworth filter at 0.3 Nyquist (0.64 $\left.\mathrm{cm}^{-1}\right)$. The SPECT data were reconstructed immediately after the scan, and the result of the SPECT scan determined. Reconstruction and region-of-interest manual delineation procedures have both been described earlier $(16,17)$.

The outcome parameter, nondisplaceable binding potential $\left(\mathrm{BP}_{\mathrm{ND}}\right)$ (18), was calculated under steady-state conditions. Reference $\mathrm{BP}_{\mathrm{ND}}$ values derived from the 28 healthy volunteers were age-corrected to match the age of the patients (using a striatal $5.6 \%$ reduction per decade (19)); a measure of the individual DAT availability was calculated according to

DAT availability $=\frac{\mathrm{BP}_{\mathrm{ND}}(\text { patient })}{\mathrm{BP}_{\mathrm{ND}}(\text { age }- \text { adjusted normal value })} \times 100 \%$.

We chose to include the lowest (right or left side) value rather than an average $\mathrm{BP}_{\mathrm{ND}}$ so that a large asymmetry would not conceal a unilaterally abnormal SPECT scan.

\section{Clinical and Diagnostic Evaluations}

Before or on the day of the SPECT scan, the patients had a routine neurologic examination, including assessment of motor features of parkinsonism (bradykinesia, tremor, rigidity, and postural stability) using the Hoehn and Yahr (H\&Y) score and assessment of global cognitive functions using the Mini Mental State Examination (MMSE).

The presence of visual hallucinations and fluctuations during the time up to the SPECT DAT scan was assessed on the basis of an interview and a review of the patient's clinical record and then was dichotomized into present or absent.

After the SPECT DAT scan, each patient was followed clinically at the Department of Neurology and a final diagnosis was determined on the basis of history, clinical symptoms, and paraclinical data. A DLB specialist determined the final clinical diagnosis between August 2008 and January 2010 in accordance with the established criteria of McKeith et al. $(2,20)$.

The result of the SPECT DAT scan was used as a supportive criterion in the diagnosis, but patients with normal DAT SPECT findings were not excluded if the criteria of probable DLB were otherwise met.

\section{Statistical Analysis}

All statistical analyses were performed with Instat 3.1a (GraphPad Software Inc.), and graphical presentations were prepared with Prism $5.00 \mathrm{v}$ (GraphPad Software Inc.). Clinical core symptoms and DAT binding were compared using the unpaired Student $t$ test. $P$ values of less than 0.05 were considered statistically significant. Correlation of continuous data was derived using linear regression analysis.

\section{RESULTS}

All patients enrolled in this study were followed for an average of $16.0 \pm 11.6$ mo (a minimum of 3 mo was required). Only patients who met the criterion of having probable DLB at followup were included in the study cohort (2). Table 1 summarizes the demographics of the cohort.

The average $\mathrm{BP}_{\mathrm{ND}}$ as a percentage of 28 age-matched healthy controls was $43.2 \% \pm 19.7 \%$ in the striatum, $48.1 \% \pm 20.0 \%$ in the caudate nucleus, and $38.3 \% \pm 24.0 \%$ in the putamen. The putamen was significantly more affected than the caudate nucleus (paired Student $t$ test, $P<0.0001$ ). Three DLB patients had normal DAT scan findings.

Figure 1 shows the cognitive dysfunction as assessed with the MMSE score versus striatal DAT binding; there was no significant correlation between MMSE score and DAT binding in the striatum, caudate nucleus, or putamen (linear regression: $P=0.41$, $P=0.24$, and $P=0.30$, respectively). Nor was there a statistically significant difference between age-corrected DAT binding and the different H\&Y scores found (Kruskal-Wallis test: $P=0.29$; Fig. 2). In many of these patients, we found it difficult to get a reliable Unified PD Rating Scale (UPDRS) motor score because of cognitive problems; that is, they did not understand how to perform the test. In addition it was difficult to address whether the patients

\section{TABLE 1}

Demographic and Clinical Data of 51 DLB Patients Included in Study

\begin{tabular}{lc}
\hline \multicolumn{1}{c}{ Parameter } & Data \\
\hline Age (mean \pm SD) $(y)$ & $74.5 \pm 8.1$ \\
Sex & $60 \%$ \\
Men & $40 \%$ \\
Women & \\
Subjective symptoms before scan & \\
(mean \pm SD) (mo) & $65.4 \pm 17.0$ \\
Duration of cognitive problems & $25.1 \pm 19.7$ \\
Duration of Parkinson core motor & \\
symptoms & \\
Symptoms on day of scan & $21.8 \pm 5.1$ \\
MMSE (mean \pm SD) (maximum, 30) & $45 \%$ \\
Tremor & $59 \%$ \\
Rigidity & $78 \%$ \\
Bradykinesia & $55 \%$ \\
Postural instability & $10 \%$ \\
No PD motor core symptoms & $65 \%$ \\
Visual hallucinations & $59 \%$ \\
REM sleep disturbances & $55 \%$ \\
Fluctuations & $2.4 \pm 0.9$ \\
\hline H\&Y (mean \pm SD) (0-5) &
\end{tabular}




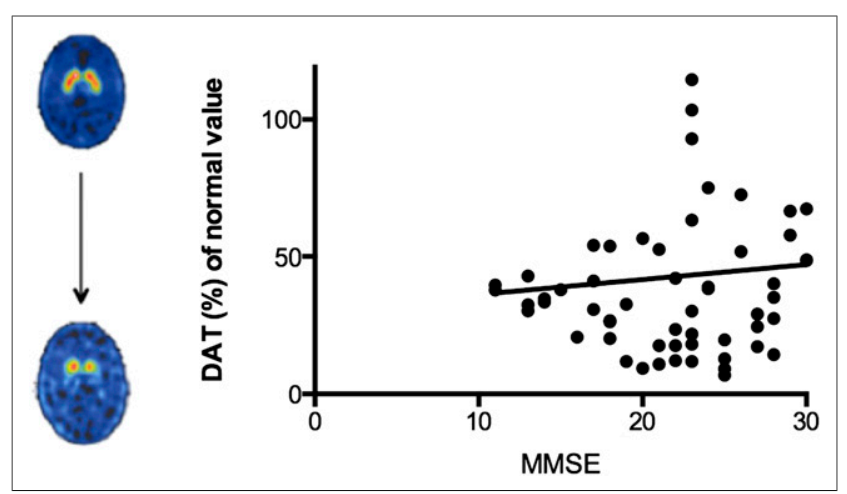

FIGURE 1. Correlation between MMSE score and lowest striatal DAT binding in percentage of age-matched expected value. Linear regression showed no significant correlation $(P=0.41)$.

had bradykinesia or "mental slowness" due to dementia. In the 10 patients tested for UPDRS motor score, we did not find a correlation with DAT availability (linear regression: $P=0.2$ ).

The demographic table illustrates a high percentage of patients presenting with fluctuations and hallucinations at the time of the initial DAT SPECT scan. To evaluate any association between either of these core features of DLB (2) and striatal neurodegeneration assessed with DAT SPECT imaging, we analyzed the patients by grouping them into those with or without hallucinations or fluctuations. There was no significant difference in the degree of parkinsonism assessed with the H\&Y score between those with and those without symptoms ( \pm hallucinations, $P>$ 0.25 ; \pm fluctuations, $P>0.25$ ). The following main analyses showed no significant difference in DAT binding in the striatum, caudate nucleus, or putamen (unpaired Student $t$ test: \pm fluctuations, $P>0.5 ; \pm$ hallucinations, $P>0.5$ ). The result of the distribution of individual $\mathrm{BP}_{\mathrm{ND}}$ values between the groups can be seen in Figure 3.

In post hoc calculations, we repeated the calculations as above, excluding the data of the 3 patients with normal DAT SPECT findings. However, the statistical outcome did not change. Finally, all main results were recalculated using $\mathrm{BP}_{\mathrm{ND}}$ values without applying the age correction, but the results were the same, and no associations were found.

\section{DISCUSSION}

In this study, we aimed to investigate the correlation between striatal dopaminergic function, assessed with DAT using ${ }^{123}$ I-PE2I SPECT imaging, and both the core features and the central features of DLB. We did not find any significant associations. The cohort of patients examined was drug-naïve, and the clinical follow-up - with an average duration of 16 mo-ensured a high clinical diagnostic accuracy of probable DLB. Even though the patients were drug-naïve, clinical severity ranged from unilateral weak symptomatic to wheelchair-bound. Further, DAT availability was sufficiently gaussian-distributed close to the mean values in the compared groups, making floor effects in the image data less likely. Therefore, the distribution of data cannot explain the lack of correlations.

Since more than half of DLB patients clinically and neuropathologically show overlap with both Alzheimer disease patients and idiopathic PD patients (21), we were interested in determining whether the cognitive dysfunction (assessed with MMSE) in DLB patients correlated with striatal dopaminergic function. Our results suggest that the general level of cognitive impairment in DLB patients is not influenced by striatal dopaminergic dysfunction. To our knowledge, only a single study (22) so far has directly addressed this issue by comparing DAT density in PD patients with and without dementia. However, that study could not find any significant difference, as supports our findings together with the fact that multiple studies have failed in discriminating between DLB and PD with DAT SPECT (9). We were not able to get information on the cognitive functions from more sensitive and extensive neuropsychologic test batteries, since this study was retrospective. We acknowledge that MMSE may be rather insensitive to the cognitive dysfunction seen in DLB. However, in a subset of subjects $(n=33)$, the Addenbrooke Cognitive Examination (23) was also performed, and we found no correlation between this test and DAT density.

We did not find any statistical difference in DAT uptake between different groups of DLB patients according to severity of parkinsonism as assessed with the H\&Y scale (Fig. 2).

In well-diagnosed PD patients, that is, patients with at least $3 \mathrm{y}$ of disease history, the severity of the disease (as assessed with, for instance, the UPDRS motor score) often correlates inversely with DAT density measured with SPECT $(8,24)$ and therefore the results are contradictive, since DLB patients demonstrate the same severity of parkinsonism as do PD patients (25). However, in theory this association could be different in DLB patients. It seems that there is a difference in the predilection of DAT SPECT tracer uptake in the basal ganglia between PD and DLB patients, with DLB patients showing a significantly lower caudate nucleus-toputamen ratio $(9,14)$. Thus, basal ganglia pathology might, after all, differ between DLB and PD patients-results that are supported by Walker et al. (9). Further, in DLB patients, multifactorial pathology could affect the nigrostriatal connection without affecting DAT binding. This effect was observed in a study by Liu et al. in which parkinsonism was induced by deposition of tangles in the basal ganglia (26). Conversely, Some DLB patients had abnormal DAT results even though they did not experience Parkinson motor symptoms. Similar results have previously been shown by McKeith et al. (27). These results are not surprising since studies of PD patients with hemiparkinsonism show presymptomatic changes in the striatum, in accordance with a 50\%-80\% loss of nigrostriatal neurons preceding symptoms (28). In this context, it is

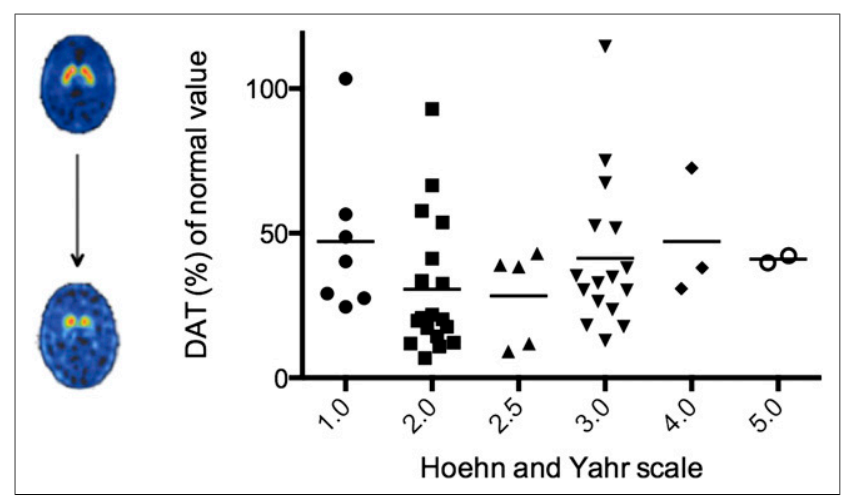

FIGURE 2. H\&Y stage distribution of striatal DAT binding in percentage of age-matched expected value. Kruskal-Wallis test did not show any significant difference between groups $(P=0.29)$. Error bars show mean. 


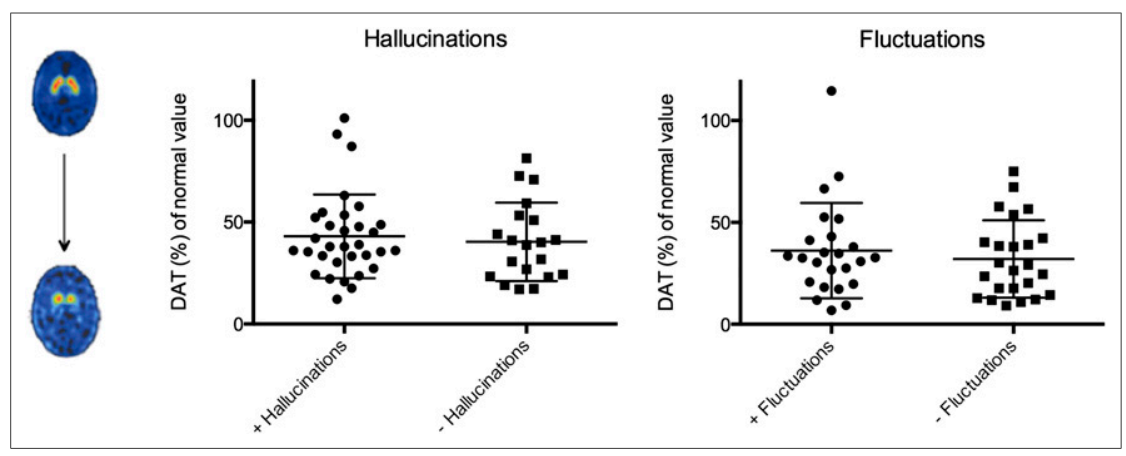

FIGURE 3. Comparison of DLB patients with and without hallucinations (left) and fluctuations (right). There was no significant difference in $\mathrm{BP}_{\mathrm{ND}}$ expressed as percentage of ageexpected value for either symptom (Student 2-tailed u2npaired $t$ test: $P=0.64 ; P=0.50$ ). Error bars show mean.

worth mentioning that neither UPDRS nor H\&Y is PD-specific to dopamine loss as is SPECT DAT imaging. UPDRS and H\&Y are scales intended to provide a comprehensive, but efficient and flexible, means to monitor PD-related disability and impairment (29). Thus, the variation in DAT reduction, at the time when symptoms appear, may explain why Parkinson motor symptoms should not correlate with DAT loss.

Signs of dementia and hallucinations play a more prominent role in the clinical picture for DLB patients than for PD dementia patients $(9,30)$. Roselli et al. showed a high inverse correlation between the degree of hallucinations and striatal function assessed with ${ }^{123}$ I-FP-CIT (12). This finding is somewhat contradictory to the literature regarding the pathophysiology for hallucinations in DLB patients. A relationship has been shown not toward the dopamine system but rather toward the nicotinic acetylcholine receptor system, with lower nicotinic receptor binding being associated with more hallucinations (31).

We did not confirm the findings of Roselli et al. (12), as we found no correlation between low striatal binding and the presence of hallucinations. There are some differences between our study and the study by Roselli et al. Primarily, ${ }^{123}$ I-FP-CIT binds not only to DAT but also to the serotonin transporter, which also is present in the striatum (13), and this factor is not discussed by Roselli et al. (13). Second, the DLB of the patients in our study was apparently at a much earlier stage (MMSE, 21.8 vs. 15.6). Finally, we included approximately 3 times as many patients in our study as did Roselli et al. (12). We did not examine the degree of hallucinations but only assessed these as present or absent on the basis of history and interviews. Thus, the data on hallucinations may be biased.

Fluctuations in cognitive functions are not specific for DLB patients and are also seen in healthy elderly individuals (32). However, in DLB patients the fluctuations are very characteristic (daytime drowsiness, lethargy, daytime sleep of 2 or more hours, staring into space for long periods, and episodes of disorganized speech) (32). So far, no studies have reported finding a correlation between striatal dopamine function and fluctuations. Impairment in the dorsolateral circuit induces problems with concentration $(11,33)$, and because problems with concentration can be regarded as fluctuations in attention, dopaminergic dysfunction in the circuit could theoretically explain fluctuations in DLB. Again, we did not find any correlation between fluctuations and ${ }^{123}$ I-PE2I binding. One reason that we did not find any correlation could be that we did not use a cognitive fluctuation scale, as was evaluated in
2000 by Walker et al. (34). In our study $55 \%$ experienced fluctuations, in line with the prevalence in other studies $(32,35)$.

A limitation of our study is that the final clinical diagnosis was not determined histopathologically. The accuracy of the clinical diagnosis was ensured by clinical follow-up only. However, the histopathologic diagnosis may also be uncertain, as DLB often presents in coexistence with Alzheimer disease and vascular dementia pathology (36). Further, there is an excellent correlation between initial DAT imaging and diagnosis by autopsy in DLB patients (37).

Even though we should be careful about drawing any major conclusions from data based on the present group of DLB patients, the results overall suggest that DLB in its early stages is more similar to Alzheimer disease with cortical dysfunction than to striatal dysfunction expressed as decreased DAT density (as in PD), and this possibility may explain the symptomatology (10). Most likely, however, the cortical dysfunction is more related to a cortical distribution of Lewy bodies than to Alzheimer disease pathology alone (21). The results of this study also suggest that in DLB, the overall striatal dopaminergic function may not have any correlation to the clinical picture since neither core features nor MMSE correlates with DAT binding, at least not when assessed by DAT SPECT imaging.

\section{CONCLUSION}

In this cohort of drug-naïve DLB patients, striatal dopaminergic function, as assessed with DAT SPECT imaging and the highly DAT-selective radioligand ${ }^{123}$ I-PE2I, did not correlate with MMSE score or with the clinical core features of DLB (parkinsonism, hallucinations, and fluctuations).

\section{DISCLOSURE}

The costs of publication of this article were defrayed in part by the payment of page charges. Therefore, and solely to indicate this fact, this article is hereby marked "advertisement" in accordance with 18 USC section 1734. This work was supported by Rigshospitalet, the Lundbeck Foundation, the EC-FP7-project, EURIPIDES HEALTH-F5-2007-201380, the Toyota Foundation, the University of Copenhagen, and HR (The Capital Region of Denmark). No other potential conflict of interest relevant to this article was reported.

\section{ACKNOWLEDGMENTS}

We thank Glenna Skouboe and Svitlana Olsen.

\section{REFERENCES}

1. Tofaris GK, Spillantini MG. Alpha-synuclein dysfunction in Lewy body diseases. Mov Disord. 2005;20(suppl 12):S37-S44.

2. McKeith IG, Dickson DW, Lowe J, et al. Diagnosis and management of dementia with Lewy bodies: third report of the DLB Consortium. Neurology. 2005;65: 1863-1872.

3. Bernheimer H, Birkmayer W, Hornykiewicz O, Jellinger K, Seitelberger F. Brain dopamine and the syndromes of Parkinson and Huntington: clinical, morphological and neurochemical correlations. J Neurol Sci. 1973;20:415-455. 
4. Hughes AJ, Daniel SE, Kilford L, Lees AJ. Accuracy of clinical diagnosis of idiopathic Parkinson's disease: a clinico-pathological study of 100 cases. J Neurol Neurosurg Psychiatry. 1992;55:181-184.

5. Sossi V, Dinelle K, Topping GJ, et al. Dopamine transporter relation to levodopaderived synaptic dopamine in a rat model of Parkinson's: an in vivo imaging study. J Neurochem. 2009;109:85-92.

6. Ashkan K, Wallace BA, Mitrofanis J, et al. SPECT imaging, immunohistochemical and behavioural correlations in the primate models of Parkinson's disease. Parkinsonism Relat Disord. 2007;13:266-275.

7. Bezard E, Dovero S, Prunier C, et al. Relationship between the appearance of symptoms and the level of nigrostriatal degeneration in a progressive 1-methyl4-phenyl-1,2,3,6-tetrahydropyridine-lesioned macaque model of Parkinson's disease. J Neurosci. 2001;21:6853-6861.

8. Benamer HT, Patterson J, Wyper DJ, Hadley DM, Macphee GJ, Grosset DG. Correlation of Parkinson's disease severity and duration with ${ }^{123}$ I-FP-CIT SPECT striatal uptake. Mov Disord. 2000;15:692-698.

9. Walker Z, Costa DC, Walker RW, et al. Striatal dopamine transporter in dementia with Lewy bodies and Parkinson disease: a comparison. Neurology. 2004;62: 1568-1572.

10. Colloby SJ, Fenwick JD, Williams ED, et al. A comparison of ${ }^{99 \mathrm{~m}} \mathrm{Tc}-\mathrm{HMPAO}$ SPET changes in dementia with Lewy bodies and Alzheimer's disease using statistical parametric mapping. Eur J Nucl Med Mol Imaging. 2002;29:615622.

11. Burruss JW, Hurley RA, Taber KH, Rauch RA, Norton RE, Hayman LA. Functional neuroanatomy of the frontal lobe circuits. Radiology. 2000;214:227-230.

12. Roselli F, Pisciotta NM, Perneczky R, et al. Severity of neuropsychiatric symptoms and dopamine transporter levels in dementia with Lewy bodies: A ${ }^{123}$ I-FPCIT SPECT study. Mov Disord. 2009;24:2097-2103.

13. Ziebell M, Holm-Hansen S, Thomsen G, et al. Serotonin transporters in dopamine transporter imaging: a head-to-head comparison of dopamine transporter SPECT radioligands ${ }^{123}$ I-FP-CIT and ${ }^{123}$ I-PE2I. J Nucl Med. 2010;51:18851891.

14. Ziebell M, Andersen BB, Thomsen G, et al. Predictive value of dopamine transporter SPECT imaging with $\left[{ }^{123} \mathrm{I}\right] \mathrm{PE} 2 \mathrm{I}$ in patients with subtle parkinsonian symptoms. Eur J Nucl Med Mol Imaging. 2012;39:242-250.

15. Pinborg LH, Ziebell M, Frokjaer VG, et al. Quantification of ${ }^{123}$ I-PE2I binding to dopamine transporter with SPECT after bolus and bolus/infusion. J Nucl Med. 2005;46:1119-1127.

16. Ziebell M, Pinborg LH, Thomsen G, et al. MR imaging-guided region-of-interest delineation is comparable to manual delineation in dopamine transporter SPECT quantification in patients: a reproducibility study. J Nucl Med Technol. 2010;38: 61-68.

17. Ziebell M, Thomsen G, Knudsen GM, et al. Reproducibility of [ ${ }^{123}$ I]PE2I binding to dopamine transporters with SPECT. Eur J Nucl Med Mol Imaging. 2007;34:101-109.

18. Innis RB, Cunningham VJ, Delforge J, et al. Consensus nomenclature for in vivo imaging of reversibly binding radioligands. J Cereb Blood Flow Metab. 2007;27:1533-1539.

19. Thomsen G, Ziebell M, Jensen PS, Knudsen GM, Pinborg LH. No correlation between body mass index and striatal dopamine transporter availability in healthy volunteers using SPECT and $\left[{ }^{123}\right.$ I]PE2I. Obesity (Silver Spring). In press.
20. Dubois B, Feldman HH, Jacova C, et al. Research criteria for the diagnosis of Alzheimer's disease: revising the NINCDS-ADRDA criteria. Lancet Neurol. 2007;6:734-746.

21. Ballard C, Ziabreva I, Perry R, et al. Differences in neuropathologic characteristics across the Lewy body dementia spectrum. Neurology. 2006;67:1931-1934.

22. Song IU, Kim YD, Cho HJ, Chung SW, Chung YA. An FP-CIT PET comparison of the differences in dopaminergic neuronal loss between idiopathic Parkinson disease with dementia and without dementia. Alzheimer Dis Assoc Disord. 2013;27:51-55.

23. Mathuranath PS, Nestor PJ, Berrios GE, Rakowicz W, Hodges JR. A brief cognitive test battery to differentiate Alzheimer's disease and frontotemporal dementia. Neurology. 2000;55:1613-1620.

24. Morrish PK. How valid is dopamine transporter imaging as a surrogate marker in research trials in Parkinson's disease? Mov Disord. 2003;18(suppl 7):S63-S70.

25. Burn DJ, Rowan EN, Minett T, et al. Extrapyramidal features in Parkinson's disease with and without dementia and dementia with Lewy bodies: a crosssectional comparative study. Mov Disord. 2003;18:884-889.

26. Liu Y, Stern Y, Chun MR, Jacobs DM, Yau P, Goldman JE. Pathological correlates of extrapyramidal signs in Alzheimer's disease. Ann Neurol. 1997;41: 368-374.

27. McKeith I, O'Brien J, Walker Z, et al. Sensitivity and specificity of dopamine transporter imaging with ${ }^{123}$ I-FP-CIT SPECT in dementia with Lewy bodies: a phase III, multicentre study. Lancet Neurol. 2007;6:305-313.

28. Marek KL, Seibyl JP, Zoghbi SS, et al. $\left[{ }^{123} \mathrm{I}\right]$ beta-CIT/SPECT imaging demonstrates bilateral loss of dopamine transporters in hemi-Parkinson's disease. Neurology. 1996;46:231-237.

29. Movement Disorder Society Task Force on Rating Scales for Parkinson's Disease. The Unified Parkinson's Disease Rating Scale (UPDRS): status and recommendations. Mov Disord. 2003;18:738-750.

30. Aarsland D, Andersen K, Larsen JP, Lolk A, Kragh-Sorensen P. Prevalence and characteristics of dementia in Parkinson disease: an 8-year prospective study. Arch Neurol. 2003;60:387-392.

31. Court JA, Ballard CG, Piggott MA, et al. Visual hallucinations are associated with lower alpha bungarotoxin binding in dementia with Lewy bodies. Pharmacol Biochem Behav. 2001;70:571-579.

32. Ferman TJ, Smith GE, Boeve BF, et al. DLB fluctuations: specific features that reliably differentiate DLB from AD and normal aging. Neurology. 2004;62: 181-187.

33. Mega MS, Cummings JL. Frontal-subcortical circuits and neuropsychiatric disorders. J Neuropsychiatry Clin Neurosci. 1994;6:358-370.

34. Walker MP, Ayre GA, Cummings JL, et al. The Clinician Assessment of Fluctuation and the One Day Fluctuation Assessment Scale: two methods to assess fluctuating confusion in dementia. Br J Psychiatry. 2000;177:252-256.

35. Farina E, Baglio F, Caffarra P, et al. Frequency and clinical features of Lewy body dementia in Italian memory clinics. Acta Biomed. 2009;80:57-64.

36. Merdes AR, Hansen LA, Jeste DV, et al. Influence of Alzheimer pathology on clinical diagnostic accuracy in dementia with Lewy bodies. Neurology. 2003;60: 1586-1590.

37. Walker Z, Jaros E, Walker RW, et al. Dementia with Lewy bodies: a comparison of clinical diagnosis, FP-CIT single photon emission computed tomography imaging and autopsy. J Neurol Neurosurg Psychiatry. 2007;78:1176-1181. 